\title{
Organizações matemática e didática entre duas coleções didáticas sobre equações do primeiro grau
}

\author{
Organizations and teaching mathematics teaching between two collections on \\ equations of the first degree
}

Edelweis Jose Tavares Barbosa edelweisb@yahoo.com.br

Anna Paula Avelar Brito Lima apbrito@gmail.com

\begin{abstract}
Resumo
Este artigo analisou a introdução do conceito de equação do primeiro grau em duas coleções de livros didáticos brasileiros do Ensino Fundamental, sendo uma escrita anterior à criação do Programa Nacional do Livro Didático (PNLD) e outra aprovada no PNLD de 2014, ambas das mesmas autoras. A Teoria Antropológica do Didático (TAD), proposta por Yves Chevallard e colaboradores, norteou teoricamente nossa pesquisa. No presente capítulo discutimos tal referencial bem como o delineamento metodológico proposto para a análise dos dados. Os resultados indicam que as organizações existentes nessas coleções nem sempre são feitas de forma a esclarecer as diferenças existentes entre os subtipos de tarefas trabalhadas, como as potencialidades das técnicas organizadas ou sistematizadas. Além destes, as coleções não alteraram as praxeologias matemáticas, mas sim as praxeologias didáticas.
\end{abstract}

Palavras-chave: Análise de Livro Didático de Matemática; Equação do Primeiro Grau; TAD

\begin{abstract}
This article analyzed the introduction, discusses the introduction of the first degree equation concept within two Brazilian Fundamental level textbook collections, as one written before the creation of the National Program of Didactical Book and the other approved in the 2011 National Program, both from the same authors. The Didactical Anthropology Theory (DAT), proposed by Yves Chevallard and collaborators, based our research theoretically speaking. In the present chapter we discuss such framework as well as the methodological proposed frame for the data analysis. The results show that the existent organizations in the collections are not done in a way to clarify the existent differences between the subtypes of activities worked, as the potentialities of the organized techniques or systematized. Moreover, the collections did not alter the mathematical praxeologies, but the didactical praxeologies.
\end{abstract}

Keywords: Mathematical Textbook Analisys ; First Degree Equation ; DAT

\section{Introdução}

Em nossa experiência em sala de aula um dos aspectos que caracteriza o início do estudo da introdução formal da Álgebra é o estudo das equações e, consequentemente, a utilização de letras para representar valores desconhecidos. Quando as letras representam valores desconhecidos, elas são usualmente denominadas de incógnitas. Entretanto, no decorrer das séries subsequentes, as letras têm outros atributos. Assim, analisamos duas coleções de livros didáticos brasileiros do Ensino Fundamental, um aprovado no Programa Nacional do Livro 
Didático (PNLD) e a outra anterior ao PNLD. Para isso, tomamos como referencial a Teoria Antropológica do Didático de Yves Chevallard, a qual parece responder com mais eficácia nossa questão de pesquisa, isto é, introdução do conceito de equação do primeiro grau presente nas duas coleções.

No ambiente escolar existe a ideia de que a Aritmética trata de números e a Álgebra de letras. Tenta-se também estabelecer limites entre conteúdos, sendo que no currículo da escola a Aritmética é trabalhada desde a educação infantil até o $6^{\circ}$ ano do Ensino Fundamental e os conteúdos tradicionais da Álgebra, tais como equações, cálculo com letras, expressões algébricas, são abordados a partir do $7^{\circ}$ ano do Ensino Fundamental, além de considerar que os conteúdos aritméticos são conhecimentos prévios para a introdução da Álgebra.

Partimos do princípio de que, segundo considera Chevallard (1998), não existe um mundo institucional ideal no qual as atividades humanas sejam geridas por praxeologias bem apropriadas que permitam realizar todas as tarefas desejadas de uma maneira eficaz, segura e inteligente. As praxeologias envelhecem na medida em que seus elementos (tipos de tarefas, técnicas, tecnologias ou teorias) perdem seus créditos ou tornam-se opacos, dando origem à constituição de novas praxeologias, necessárias ao melhor funcionamento de uma determinada instituição, em consequência dos novos tipos de tarefas (tipos de problemas) que se apresentam a essa instituição.

Sendo assim, apresentamos o artigo em duas seções. A primeira com relação à fundamentação teórica, modelização a priori, seleção e caracterização das obras analisadas. A segunda seção discute os principais resultados e algumas considerações. Álgebra escolar: aspectos

\section{históricos e concepções}

Traduzindo de forma literal o título do livro de Al-Khowarizmi encontramos a ciência da restauração (ou reunião) e redução. Matematicamente seria melhor ciência da transposição e do cancelamento, ou ainda "a transposição de termos subtraídos para o outro membro da equação e o cancelamento de termos semelhantes (iguais) em membros opostos da equação" (BOYER, 1996, p.156).

No desenvolvimento da Álgebra observa-se que desde civilizações antigas do Egito e Babilônia até os dias atuais a linguagem matemática evolui gradativamente, passando por várias fases que marcaram época. Os historiadores dividem a história da Álgebra em três principais fases: retórica ou verbal, sincopada e simbólica (GUELLI, 2005; BOYER, 1996): 
Álgebra Retórica (ou verbal) - A fase retórica ou verbal se estende desde os Babilônios (1700 a.C.) até o matemático grego Diofanto (250 d.C.). É caracterizada pela completa ausência de símbolos e abreviações que possam expressar o pensamento algébrico; todos os passos relativos a números e equações eram descritos na linguagem corrente. Esta teria sido a Álgebra dos Egípcios, dos Babilônios e dos gregos pré-diofantinos;

Álgebra Sincopada - Essa fase teria surgido com Diofanto de Alexandria, e ficado marcada pela introdução de um símbolo para a incógnita, utilizando uma forma mais abreviada e concisa para expressar suas equações. É registrada também na história uma sincopada similar à de Diofanto, que surgiu por meio dos Hindus, especialmente por Brahmagupta (século XII). Essa fase se prolongou até o início do século XVI. Neste momento histórico temos a impressão de que os matemáticos não demorariam muito tempo para descobrirem os sinais; e, Álgebra Simbólica - Os registros indicam que essa fase teve seu inicio a partir do momento em que as ideias algébricas passaram a ser expressas somente através de símbolos, deixando de lado o uso das palavras. Embora o jurista francês François Viète (1540- 1603), ainda utilizasse um estilo sincopado, foi ele o principal responsável pela criação de novos símbolos na Álgebra.

No Brasil, Fiorentini, Miorim e Miguel (1993) fizeram uma abordagem histórica e evidenciam três concepções de educação algébrica que vêm exercendo maior influência no Ensino de Matemática elementar.

A primeira, chamada de linguístico-pragmática, baseia-se no papel do Ensino da Álgebra buscando fornecer um instrumental técnico (superior ao da Aritmética) para a resolução de equações ou de problemas equacionáveis. Para o aluno adquirir essa capacidade considera-se necessário e suficiente primeiro dominar, ainda que de forma mecânica, as técnicas requeridas pelo transformismo algébrico (sintaxe).

O currículo de Ensino da Álgebra tem, portanto, como ponto de partida, o cálculo literal (operações de adição, subtração, multiplicação/fatoração e divisão de expressões algébricas), o qual é desenvolvido por meio de muitos exercícios visando capacitar os alunos no manejo preciso dessas expressões algébricas. Só depois disso é que são introduzidos problemas do tipo aplicação algébrica.

Os mesmos autores apresentam a segunda concepção, Fundamentalista-estrutural, que surge aproximadamente, na segunda metade do século XX, predominantemente nas décadas de 1970 e 1980, e vem contrapor à ideia anterior com um cunho fundamentalista. O papel do 
Ensino da Álgebra seria o de fornecer os fundamentos lógico-matemáticos para toda a Matemática escolar, inclusive aqueles tradicionalmente considerados algébricos, como o cálculo algébrico e o estudo das equações. Isto é realizado por meio da introdução dos campos numéricos, da Teoria dos Conjuntos, das estruturas e das propriedades (fechamento, comutativa, elemento neutro,...), das relações e funções. Assim, o emprego das propriedades estruturais das operações serve para justificar logicamente cada passagem presente no transformismo algébrico.

A terceira concepção, Fundamentalista-analógica, é uma síntese das duas anteriores, pois tenta recuperar o valor instrumental da Álgebra e preserva a preocupação fundamentalista, não mais com base nas propriedades estruturais, por meio do uso de modelos analógicos geométricos (blocos de madeira ou mesmo figuras geométricas) ou físicos (como a balança) que visualizam ou justificam as passagens do transformismo algébrico. A Álgebra geométrica era didaticamente superior a qualquer outra abordagem lógico-simbólica, pois tornam visíveis certas identidades algébricas.

O ponto problemático e comum entre essas três concepções, segundo Fiorentini, Miorim e Miguel (1993), é que elas praticamente reduzem o Ensino da Álgebra aos seus aspectos linguísticos e transformistas, dando mais ênfase à sintaxe da linguagem algébrica que ao pensamento algébrico e seu processo de significação (a semântica).

As três concepções enfatizam o Ensino de uma linguagem algébrica já constituída, priorizando o domínio, por parte do aluno, de habilidades manipulativas das expressões algébricas. Além disso, a Álgebra não se reduz a um instrumento técnico-formal que facilita a resolução de certos problemas.

De acordo com Miguel, Fiorentini e Miorim (1992), desde 1799, momento em que a Álgebra passa a fazer parte do currículo no Brasil, até o início da década de 1960 prevaleceu um Ensino de caráter reprodutivo, sem clareza, no qual tudo era essencial. A matemática escolar apresentava-se dividida em compartimentos estanques. Primeiro estudava-se a Aritmética, depois a Álgebra e, em seguida, a Geometria. Neste período, segundo os autores, a Álgebra apresentava um caráter mais instrumental, útil apenas para resolver equações e problemas.

Miguel, Fiorentini e Miorim (1992) ressaltam o fato de que a Álgebra pós Matemática Moderna parece retomar seu papel, anteriormente ocupado, ou seja, de um estudo com a finalidade de resolver equações e problemas. Tentou-se recuperar seu valor instrumental, mantendo seu caráter fundamentalista. Os autores destacam ainda que a Álgebra, apesar de 
ocupar boa parte dos livros didáticos atuais, não tem recebido a devida atenção nos debates, estudos e reflexões a respeito do Ensino da Matemática. Álgebra no currículo da educação básica

Educadores demonstram preocupação com a compreensão da Álgebra no ensino da Matemática, e especialmente as noções que devem ser trabalhadas para a compreensão do que venha a ser uma equação (BRITO MENEZES, 2006). De acordo com os Parâmetros Curriculares Nacionais (BRASIL, 1998), o estudo da Álgebra constitui um espaço bastante significativo, propiciando ao aluno o desenvolvimento e o exercício de sua capacidade de abstração e generalização.

Lee (1996) propõe que a Álgebra é uma minicultura na cultura da Matemática. Nesse sentido, existem concepções e perspectivas específicas acerca desse saber que se faz necessário de serem compreendidas para que possamos ter uma noção da dimensão e do potencial da Álgebra, sobretudo focalizando o seu ensino (BRITO MENEZES, 2006).

Lins e Gimenez (1997) caracterizam a Álgebra como sendo um conjunto de afirmações para as quais é possível produzir significado em termos de números e operações aritméticas, possivelmente envolvendo igualdade ou desigualdade.

Segundo Souza e Diniz (1996), a Álgebra é a linguagem da Matemática utilizada para expressar fatos genéricos. Como toda linguagem, a Álgebra possui seus símbolos e suas regras. Para Garcia (1997) a Álgebra revoluciona por ser uma ferramenta a serviço da resolução de problemas e ser um objeto matemático em si, um ramo autônomo das Matemáticas de que todas as disciplinas científicas se nutrem para estabelecer melhores e mais cômodas vias de comunicação entre elas e com o exterior.

Entretanto, as atividades propostas pelos educadores seguem em caminhos contrários, isto é nas orientações para o trabalho com os problemas algébricos é dada ênfase puramente ao processo de resolução. Fazer o aluno pensar, questionar fica em segundo plano, tornando estas atividades puramente mecânicas, rotineiras e muitas vezes desinteressantes para o mesmo.

Neste contexto, Germi (1997) destaca três diferentes status possíveis para as letras na Matemática escolar:

1. Para Designar: As letras servem para designar uma dimensão (largura, comprimento, altura, etc.) nas fórmulas de cálculo de perímetro e área, ou ainda para designar 
objetos geométricos simples (pontos, retas, círculos, ângulos). A letra nessas situações é uma ferramenta de designação;

2. No cálculo Algébrico: A letra é considerada como um número desconhecido numa equação:

Neste estágio reside uma real dificuldade didática: designado um número desconhecido por uma letra nós o manipulamos, na verdade, como se ele fosse conhecido, os números que faltam são pensados como números precisos, designados provisoriamente por letras de maneira que a nossa ignorância inicial não nos impeça de fazê-los participar do cálculo (BOUJADDI, 1996, apud GERMI, 1997, p. 62).

A aparição de resoluções de equações mais complexas desencadeia a necessidade de operações elementares sobre expressões literais, portanto, as letras tomam um novo status: elas tomam então um status indeterminado, no sentido que elas não têm mais necessidade, para essas representações, de ser um número (BOUJADDI, 1996).

\section{Equações do Primeiro Grau: elementos}

O estudo de equações do $1^{\circ}$ grau com uma incógnita baseia-se na estrutura algébrica denominada anel dos polinômios a uma indeterminada. Este anel é simbolizado usualmente por $R[x] R$, representando o corpo dos números reais e consiste das expressões formais $p(x)=a_{0}+\ldots+a_{n} x^{n}, \mathrm{n}$ um número natural, no qual se definem as operações de adição de dois polinômios e de multiplicação de um polinômio por um número real, as quais se supõem, satisfazem as propriedades expressas nas regras usuais da Álgebra, (ARAÚJO, 2009, p. 35):

A operação de adição de dois polinômios $p(x)=a_{0}+a_{1} \ldots+a_{n} x^{n}$ com um polinômio $p(x)=a_{0}+\ldots+a_{n} x^{n} \quad$ com um polinômio $p(x)=b_{0}+b_{1} x \ldots+b_{n} x^{n} \quad$ é definida por: $p(x)+q(x)=p(x)=a_{0}+a_{1} \ldots+a_{n} x^{n}+p(x)=b_{0}+b_{1} x \ldots+b_{n} x^{n}=$ $\left(a_{0}+b_{0^{\prime}}\right)+\left(a_{1}+b_{1}\right) x+\ldots+\left(a_{n}+b_{n}\right) x^{n}$ e satisfaz as seguintes propriedades:

Para todo $p(x) \cdot q(x)_{--} e_{--} r(x) \mathrm{p}(\mathrm{x}) . \mathrm{q}(\mathrm{x})$ e $\mathrm{r}(\mathrm{x})$ pertencem a $R[x]$,

a) $p(x)+q(x)=p(x)+q(x)$

b) $[p(x)+q(x)]+(r)=q(x)+[p(x)+r(x)]$

c) $p(x)+0(x)=0(x)+p(x)$, em que $0(x)$ representa um polinômio nulo $0_{0}+0 x+\ldots 0 x^{n}, n \in \mathrm{N}$. 
d) Para todo $p(x)+q(x)=a_{0}+a_{1} x+\ldots a_{n} x^{n}$ existe o polinômio $p^{\prime}(x)$ tal que $p(x)+p^{\prime}(x)=0(x)$.

Sabe-se que $p^{\prime}(x)=\left(-a_{0}\right)+\left(-a_{1}\right) x \ldots+\left(-a_{n}\right) x^{n}$

A operação de multiplicação de um número real $\mathrm{K}$ por um polinômio $p(x)=a_{0}+a_{1} x+\ldots+a_{n} x^{n}$ é definida por:

$$
K p(x)=\left(K a_{0}\right)+(k a 0) x+\ldots+\left(K a_{n}\right) x^{n}
$$

e satisfaz as seguintes propriedades:

Para todo $K, K_{1}, K_{2} \in R$ e $p(x)$ e $q(x) \in R[x]$,

$$
K[p(x)+q(x)]=k p(x)+k q(x)
$$

a) $K_{1}\left[k_{2} p(x)\right]=\left(k_{1} k_{2}\right) p(x)$

b) $1 p(x)=p(x)$

O polinômio, assim definido, tem grau $n$ se $a_{n} \neq 0$. No caso em $n=1$, dizemos que $p(x)=a_{0}+a_{1} x$ tem grau 1. Nesse caso, $p(x)$ é denominado polinômio do $1^{\circ}$ grau na indeterminada $x$.

Por outro lado, para cada polinômio $p(x)=a_{0}+a_{1} \ldots+a_{n} x^{n} \in R[x]$ é possível definir uma função polinomial $f: R \rightarrow R$, indicada por $f(x)=a_{n} x^{n}+\ldots a_{0}$. A função assim definida, associada cada número $k \in R$ em $f(k) \in R$.

Se existe um número $k \in R$ tal que $f(k)=0$, dizemos que $k$ é raiz (zero) de $f(x)$. Nesse caso, para determinar as raízes do polinômio é necessário determinar os valores de $x \in R$ tal que $f(x)=0$, ou seja, $a_{n} x^{n}+\ldots+a_{0}=0$. Essa última igualdade é denominada de equação polinomial de grau $n$. No caso em que $n=1$, temos uma equação polinomial do $1^{\circ}$ grau ( $a_{0}+a_{1} x=0$ ), que é o nosso objeto de estudo. Os números reais tais que $f(\propto)=0$ são denominados soluções da equação $f(x)=0$ (ARAÚJO, 2009).

Com base nas definições anteriores, denomina-se equação do $1^{\circ}$ grau toda equação na forma $a x+b=0$, onde a incógnita possui expoente 1 . A equação do $1^{\circ}$ grau é chamada linear, pois sua representação gráfica é uma linha reta.

As operações e propriedades dos polinômios, enunciadas anteriormente, nos permitem ainda elaborar os princípios que fundamentam a resolução de equações (ARAÚJO, 2009, p. 45):

- Princípio aditivo: se adicionarmos a ambos os membros (por exemplo: $3 x+5=x-1$ antes da igualdade chamamos de $1^{\circ}$ membro e após a igualdade de segundo membro) 
de uma equação um mesmo número ou uma mesma expressão algébrica, obteremos uma equação equivalente à primeira; e,

- Princípio multiplicativo: se multiplicarmos ambos os membros de uma equação pelo mesmo número (diferente de zero) ou uma mesma expressão algébrica (não nula), obteremos uma equação equivalente à primeira.

Estes dois princípios acima são usados na elaboração de técnicas para resolver, por exemplo, equações do $1^{\circ}$ grau.

Em relação ao ensino de resoluções de equações, Bernard e Cohen (1995) recomendam um conjunto gradativo de ensino para encontrar as raízes de uma equação, sendo descrito em quatro métodos, assim denominados: (1) gerar e avaliar; (2) esconder; (3) desfazer e (4) equações equivalentes. Para estes autores, cada novo método é subsequente de resolução deriva de seu anterior, beneficiando a passagem de procedimentos aritméticos para o algébrico.

O método de gerar e avaliar incide em levar o aluno a pensar no conceito de número e a provocar diferentes valores para serem testados por tentativa e erros. Bernard e Cohen (1995) analisam que nesse de gerar e avaliar, o aluno não se limita a ficar fazendo tentativas e erros, aleatoriamente. Para estes autores, intuitivamente, o aluno segue um esquema de cálculo de valores que se realimenta no processo de geração de valores.

O método de esconder consiste em levar o aluno a resolver a equação pensando sobre o ela pede. Por exemplo, a equação $10-\mathrm{x}=6$ esconde-se o $x$ e pergunta-se que número devemos subtrair de 10 para 6? Os autores consideram que este método permite chegar a uma conceituação mais ampla de incógnita, levando o aluno a perceber que uma expressão pode ser uma incógnita.

O método de desfazer fundamenta-se na noção de operações inversas e na reversibilidade de um processo, envolvendo um ou mais passos invertíveis. Desse modo, o aluno deve ser orientado a raciocinar sobre o que esta acontecendo operacionalmente com uma incógnita e criar uma sequência de perguntas dirigidas sobre como voltar ao ponto de partida, isto é, a incógnita. Assim por exemplo, no caso da equação $\frac{7(2 x-3)-5}{10}=5$. 
Figura 1: Modelo gerado pelo método de desfazer

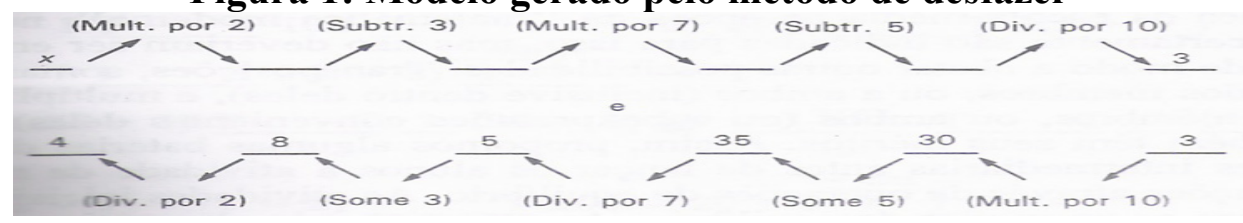

Fonte: Bernard e Cohen (1995, p.117)

Esse procedimento de voltar ao ponto de partida, utilizando apenas cálculo aritmético, estimula o aluno a desenvolver a reversibilidade, a análise e a resolução de problema.

O método de equações equivalentes fundamenta-se em efetuar operações de equilíbrio nos dois membros da igualdade (somando um número ou expressão aos dois membros da igualdade) até que um lado esteja a incógnita e do outro, um número. As novas equações obtidas por esse processo preservam o mesmo conjunto de soluções e por isso são denominadas equações equivalentes.

\section{Teoria Antropológica do Didático}

Segundo Chevallard (1999, p.1), essa teoria estuda o homem perante o saber matemático, e mais especificamente, perante situações Matemáticas. Um motivo para utilização do termo antropológica é que a TAD situa a atividade Matemática e, em consequência, o estudo da Matemática dentro do conjunto de atividades humanas e de instituições sociais. Assim, a Teoria Antropologica do Didático (TAD) considera como elementos primitivos INSTITUIÇÕES (I), INDIVIDUOS (X) e OBJETO (O).

Chevallard (1999, p.1) considera que uma instituição (I) é um dispositivo social total que pode ter apenas uma extensão muito reduzida no espaço social, mas que permite e impõe a seus sujeitos (...) maneiras próprias de fazer e de pensar. Sob a ótica da TAD cada saber é saber de pelo menos uma instituição; um mesmo objeto do saber pode viver em instituições diferentes e para viver em uma instituição; um saber necessita submeter-se a certas imposições, o que o conduz a ser transformado.

A TAD consiste no desenvolvimento da noção de organização praxeológica que, de acordo com Chevallard, acrescenta às noções acima descritas, as noções de (tipo de) tarefa, técnica, tecnologia e teoria. Para ele, tais noções vão permitir modelizar às práticas sociais em geral as atividades Matemáticas, como descritas a seguir. 


\section{Organização Praxeológica}

Podemos entender uma organização praxeológica como a realização de certo tipo de tarefa t que se exprime por um verbo, pertencente a um conjunto de tarefas do mesmo tipo t, através de uma técnica $\tau$, justificada por uma tecnologia $\theta$, que por sua vez, é justificada por uma teoria $\Theta$. Parte do postulado que qualquer atividade humana põe em prática uma organização, denominada por Chevallard (1998) de praxeologia, simbolizada pela notação [t, $\tau, \theta, \Theta]$.

Chevallard (1998) considera ainda que o par $[\mathrm{t}, \tau]$ está relacionado à prática, e pode ser compreendido como um saber-fazer, e o par $[\theta, \Theta]$ relacionado a razão, é compreendido como o saber. O autor define assim a Organização Praxeológica $[t, \tau, \theta, \Theta]$, em que temos um bloco prático $[\mathrm{t}, \tau]$, composto das tarefas e técnicas, o chamado saber fazer, e um bloco teórico $[\theta$, $\Theta]$, composto pelas tecnologias e teorias, o bloco do saber. Considera ainda que a existência de um tipo de tarefa matemática em um sistema de ensino está condicionada à existência de, no mínimo, uma técnica de estudo desse tipo de tarefa e uma tecnologia relativa a esta técnica, mesmo que a teoria que justifique essa tecnologia seja negligenciada.

Os tipos de tarefas $(\mathrm{t})$ que se situam em acordo com o princípio antropológico supõem a existência de objetos bem precisos e que não são obtidos diretamente da natureza. Eles são artefatos, obras, construtos institucionais, como por exemplo, uma sala de aula, cuja reconstrução é inteiramente um problema, que é o objeto da didática (CHEVALLARD, 1998). Por exemplo, resolva a equação $3 x+6=30$. A noção de tarefa, ou especificamente do tipo de tarefa, tendo como um objetivo bem definido, por exemplo, encontrar o valor de x é um tipo de tarefa, mas calcular não explicita o que é calcular. Assim, calcular o valor de uma equação é um tipo de tarefa, mas somente calcular não seria um tipo de tarefa. Para esse exemplo, calcular é gênero de tarefa.

Uma técnica $(\tau)$ é uma maneira de fazer ou realizar as tarefas $\tau \in \mathrm{t}$. Segundo Chevallard (1998), uma praxeologia relativa a um tipo de tarefa t necessita, em princípio, de uma técnica $\tau$ relativa. No entanto, ele afirma que uma determinada técnica $\tau$ pode não ser suficiente para realizar todas as tarefas $\tau \in \mathrm{t}$. Ela pode funcionar para uma parte $\mathrm{p}(\tau)$ das tarefas $\mathrm{t}$ e fracassar para $t / p(\tau)$. Isso significa que em uma praxeologia pode existir uma técnica superior a outras técnicas, ao menos no que concerne à realização de certo número de tarefas de $\mathrm{t}$ (CHEVALLARD, 1998). Por exemplo, a multiplicação no conjunto dos números naturais sempre aumenta, mas que pode fracassar em outro conjunto numérico. 
A tecnologia $(\theta)$ é definida inicialmente como um discurso racional sobre uma técnica $\tau$, cujo primeiro objetivo consiste em justificá-la racionalmente, isto é, em assegurar que a técnica permita que se cumpra bem a tarefa do tipo t. Na Matemática, tradicionalmente, a justificação de uma técnica é realizada por meio de demonstração. O segundo objetivo da tecnologia consiste em explicar, tornar inteligível e esclarecer uma técnica $\tau \cdot$, isto é, em expor por que ela funciona bem. Além disso, a tecnologia tem também a função de reproduzir novas técnicas, mais eficientes e adaptadas à realização de uma determinada tarefa (CHEVALARD, 1998).

A teoria $(\Theta)$ tem como objetivos justificar e esclarecer a tecnologia, bem como tornar inteligível o discurso tecnológico. Passa-se então a um nível superior de justificaçãoexplicação- produção, [...] retomando com relação à tecnologia o papel que esta tem em relação à técnica. O autor adverte, no entanto, que geralmente essa capacidade de justificar e de explicar a teoria é quase sempre obscurecida pela forma abstrata como os enunciados teóricos são apresentados frequentemente (CHEVALLARD, 1998).

Uma organização matemática é elaborada em torno de uma noção, ou conceito, inerente à própria Matemática. As Praxeologias Matemáticas (OM) são as respostas (a rigor) a questões do tipo como realizar o estudo de determinado assunto. Refere-se ao modo que possibilita a realização do estudo de um determinado tema, o conjunto de tarefas, de técnicas, de tecnologias, entre outras, mobilizadas para o estudo de um tema. Por exemplo, encontrar o valor de uma incógnita de uma equação.

Quaisquer que sejam as escolhas adotadas nos cursos dos trabalhos de estudo de dada OM algumas situações estão necessariamente presentes, mesmo que estas se apresentem de formas variadas, tanto de forma quantitativa como qualitativamente falando. Estas situações serão denominadas de momentos de estudos, ou momentos didáticos, porque podemos dizer que qualquer que seja o caminho escolhido ele conduzirá inevitavelmente a um momento de fixação, ou de institucionalização, ou a um momento que demandará o questionamento do que é valido acerca do que foi construído, que caracteriza o momento de avaliação, dentre outros.

O primeiro momento é o primeiro encontro com a organização que está sendo estudada. $\mathrm{O}$ segundo é o da exploração do tipo de tarefas t e de elaboração de uma técnica $\tau$ relativa a este tipo de tarefas. O terceiro momento é o da constituição do ambiente tecnológico-teórico relativo à técnica. O quarto é o do trabalho da técnica que visa melhorá-la, torná-la mais confiável, o que geralmente exige aprimorar a tecnologia até então elaborada e aumentar o 
controle que se tem sobre a técnica. O quinto momento é o da institucionalização que mostra o que realmente é a OM constituída, apontando os elementos que permanecerão definitivamente na OM e os que serão dispensados.

Finalmente, o sexto momento, o da avaliação, que se articula com o momento da institucionalização e permite relançar o estudo, demanda a retomada de alguns dos momentos, e eventualmente do conjunto do trajeto didático.

\section{Analisando as Coleções}

A metodologia seguida para a caracterização, análise e comparação das organizações matemáticas e didáticas existentes sobre o ensino de equações do $1^{\circ}$ grau nessas duas coleções para Aprender Matemática (não avaliada no PNLD) e Ideias e Desafios ambas de Iracema e Dulce (aprovada no PNLD 2014) constitui-se em duas etapas de trabalho. A primeira trata-se da modelização a priori, das praxeologias matemáticas pontuais existentes em torno da resolução de equações do $1^{\circ}$ grau, ao menos em termos de subtipos de tarefas, técnicas e tecnologias, a partir de estudos teóricos e didáticos. A segunda etapa constitui-se da caracterização das obras analisadas, apresentando sua identificação, os motivos da escolha, descrição da estrutura e da forma de organização dos conteúdos.

\section{Modelização a priori}

Tomando como base estudos teóricos, encontramos basicamente dois tipos de definições para equações. A primeira, mais geral, define equação como uma igualdade que envolve uma ou mais quantidades desconhecidas (incógnitas) (CUNHA, 1887; CALADO, 1952; COSTA e DOS ANJOS, 1970); a segunda, mais específica, sobre equações do $1^{\circ}$ grau, como toda equação que se pode reduzir à forma $a x=b$, com $a, b \in R$ e $a \neq 0$ (CALADO, 1952).

Chevallard (1994) classifica os procedimentos de resoluções de equações do primeiro grau em duas categorias: (1) equações do tipo $a x+b=c$, que podem ser resolvidas por procedimentos aritméticos e (2) equações do tipo $a_{1} x+b_{1}=a_{2} x+b_{2}$, que não podem ser resolvidas por procedimentos que se apoiem especificamente em operações aritméticas. Nessa definição, $x$ é a incógnita e $a_{1}, b_{1} \in \mathfrak{R}$ com $a_{1} \neq 0$.

No entanto, nem sempre as equações do $1^{\circ}$ grau apresentam-se escritas nas formas simplificadas. Frequentemente, numa atividade, elas aparecem sob diferentes formas, dentre 
as quais destacamos outras duas categorias: equações dos tipos $A(x)=c$ e $A_{1}(x)=A_{2}(x)$, em que $A(x), A_{1}(x)$ e $A_{2}(x)$ são expressões polinomiais, na variável $x$, que ainda não foram reduzidas à forma canônica $a x+b$, e $a, b \in \mathfrak{R}$ e $a \neq 0$, mas que podem ser reduzidas a esta forma por processo de desenvolvimento e redução.

Portanto, para este estudo, classificamos e caracterizamos a priori os seguintes subtipos de tarefas relativos à resolução de equações do $1^{\circ}$ grau com uma incógnita, no campo do $\mathfrak{R}$, em quatro categorias: (1) resolver equação uma equação do tipo $a x+b=c\left(\mathrm{t}_{1}\right)$, como por exemplo, $2 x+5=15$; (2) resolver uma equação do tipo $A(x)=c$, sendo $A(x)$ uma expressão polinomial não reduzida à forma $\left(\mathrm{t}_{2}\right)$, por exemplo, $3(x+2)+x=20$; (3) resolver uma equação do tipo $a_{1} x+b_{1}=a_{2} x+b_{2}\left(\mathrm{t}_{3}\right)$, por exemplo, $2 x-4=x+5$; (4) resolver uma equação do tipo $A_{1}(x)=A_{2}(x)$, sendo $A_{1}(x)$ ou $A_{2}(x)$, expressões polinomiais não reduzidas à forma canônica $\left(\mathrm{t}_{4}\right)$, por exemplo, $2(x-3)+x=x-8$.

Para resolver tais subtipos de tarefas foram identificadas e categorizadas a priori as seguintes técnicas $(\tau)$ : a) Testar a igualdade $\left(\tau_{\mathrm{TI}}\right)$, por tentativas e erros; b) Transpor termos ou coeficientes $\left(\tau_{\mathrm{TTC}}\right)$, invertendo as operações; c) Neutralizar termos ou coeficientes $\left(\tau_{\mathrm{NTC}}\right)$, efetuando a mesma operação nos dois membros da igualdade; d) Reagrupar os termos semelhantes $\left(\tau_{\mathrm{RTS}}\right)$, invertendo o sinal dos termos transpostos.

Além dessas técnicas próprias de resoluções de equações, para os casos dos subtipos de tarefas $\tau_{2}$ e $\tau_{4}$, temos também a seguinte técnica: e) Desenvolver ou reduzir expressões $\left(\tau_{\mathrm{DRE}}\right)$, eliminando parênteses e/ou agrupando termos semelhantes. Enfim, dependendo das variáveis mobilizadas na construção das equações, podemos mobilizar uma ou mais técnicas, dando origem às técnicas mistas.

Para justificar as técnicas caracterizadas acima para resolver equações do $1^{\mathrm{o}}$ grau com uma incógnita, foram identificadas e caracterizadas a priori as seguintes tecnologias: a) Princípios de equivalência entre equações: equações com as mesmas soluções ou raízes $\left(\theta_{\mathrm{PPE}}\right)$; b) Princípio aditivo: quando aos dois membros de uma equação se adiciona (ou deles se subtrai) a mesma quantidade, obtém-se uma nova equação equivalente à primeira; c) Princípio multiplicativo: quando aos dois membros de uma equação se multiplica (ou deles se divide) a mesma quantidade (diferente de zero), obtém-se uma nova equação equivalente à primeira; $d$ ) Propriedades das operações inversas em $\mathfrak{R}$ (conjunto dos números reais) ou leis da transposição de termos $\left(\theta_{\mathrm{POI}}\right): 1$ ) Se a, b, c são números reais tais que $\mathrm{a}+\mathrm{b}=\mathrm{c}$, então $\mathrm{a}=\mathrm{b}-$ 
c; 2) Se a, b e c são números reais tais que $\mathrm{a} \cdot \mathrm{b}=\mathrm{c}$, então $\mathrm{a}=\mathrm{c} \div \mathrm{b}, \mathrm{b} \neq 0$; 3) Propriedades gerais da igualdade $\left(\theta_{\mathrm{PGI}}\right)$ ou lei do cancelamento: 1) Se $\left.a+b=a+c \Leftrightarrow b=c ; 2\right) \mathrm{Se}$ $a . b=a . c \Leftrightarrow b=c$ com $a \neq 0,3)$ Propriedades distributivas ( $\left.\theta_{\mathrm{PDM}}\right)$ : Se $k, a, b, c \mathrm{e}$ e $d$ são números reais, então $k(a+b)=k a+k b$ e $(a+b)(c+d)=a c+a d+b c+b d$.

Após apresentação e categorização das tarefas, bem como de suas tecnologias, analisamos duas coleções didáticas do $7^{\circ}$ ano do Ensino Fundamental, sendo elas Para Aprender Matemática e Ideias e Desafios, ambas de Iracema e Dulce, apresentamos os principais resultados desse estudo comparativo das organizações existentes nessas coleções.

\section{Principais resultados}

Aqui analisamos os principais resultados do estudo das organizações didáticas e das praxeologias matemáticas nessas duas coleções didáticas do $7^{\circ}$ ano, especificamente o capítulo de equações do $1^{\circ}$ grau. Desse modo, utilizamos as categorias modelizadas a priori relativas às praxeologias matemáticas relativas ao subtipo de tarefa resolver equações do primeiro grau, em termos de subtipos de tarefas, técnicas e tecnologias:

Quadro 1: Comparativo entre duas coleções quanto aos Subtipos de Tarefas

\begin{tabular}{|c|c|c|c|c|}
\hline \multirow[t]{2}{*}{$\begin{array}{l}\text { SUBTIPOS DE } \\
\text { TAREFAS }\end{array}$} & \multicolumn{2}{|c|}{$\begin{array}{c}\text { COLEÇÃO PARA } \\
\text { APRENDER } \\
\text { MATEMÁTICA }\end{array}$} & \multicolumn{2}{|c|}{$\begin{array}{c}\text { COLEÇÃO IDEIASE } \\
\text { DESAFIOS }\end{array}$} \\
\hline & TÉCNICA & $\begin{array}{c}\text { TECNOLOGI } \\
\mathrm{A}\end{array}$ & TÉCNICA & $\begin{array}{c}\text { TECNOLOGI } \\
\mathrm{A}\end{array}$ \\
\hline \multirow{3}{*}{$\mathbf{t}_{1}: \quad a x+b=c$} & $\tau_{\mathrm{TI}}$ & $\begin{array}{c}\text { Regras de } \\
\text { propriedades } \\
\text { operatórias }\end{array}$ & $\tau_{\mathrm{TI}}$ & $\begin{array}{c}\text { Regras de } \\
\text { propriedades } \\
\text { operatórias }\end{array}$ \\
\hline & \multirow[t]{2}{*}{$\tau_{\mathrm{NTC}-} \tau_{\mathrm{TTC}}$} & $\theta_{\mathrm{PEE}}$ & \multirow[t]{2}{*}{$\tau_{\mathrm{NTC}} \tau_{\mathrm{TTC}}$} & $\theta_{\mathrm{PEE}}$ \\
\hline & & $\theta_{\text {PGI PEE }}$ & & $\theta_{\text {PGI PEE }}$ \\
\hline \multirow[t]{2}{*}{$\mathbf{t}_{2}: \quad A(x)=c$} & \multirow[t]{2}{*}{$\tau_{\mathrm{TTC}}$} & $\theta_{\text {PGI_PDM }}$ & \multirow[t]{2}{*}{$\tau_{\mathrm{TTC}}$} & $\theta_{\text {PGI_PDM }}$ \\
\hline & & $\theta_{\mathrm{PGI}}$ & & $\theta_{\mathrm{PGI}}$ \\
\hline $\mathbf{t}_{3}: a_{1} x+b_{1}=a_{2} x+b_{2}$ & \multirow{2}{*}{$\begin{array}{c}\tau_{\mathrm{ED} \_\mathrm{DRE}} \\
\text { NTC }\end{array}$} & $\theta_{\text {DRE_PGI }}$ & \multirow[t]{2}{*}{$\tau_{\text {ED_DRE_NTC }}$} & $\theta_{\text {DRE_PGI }}$ \\
\hline $\mathbf{t}_{\mathbf{4}}: \quad A_{1}(x)=A_{2}(x)$ & & $\theta_{\text {DRE_PEE }}$ & & $\theta_{\text {DRE_PEE }}$ \\
\hline
\end{tabular}

Na coleção Para Aprender Matemática as relações da transposição das Praxeologias Matemáticas pontuais existentes em torno dos subtipos de tarefas referentes às resoluções de equações do $1^{\circ}$ grau ocorrem em três momentos: primeiro momento- introdução de um problema ou situação realizada para formar ou sistematizar a técnica eletiva para resolver a 
equação (subtipo de tarefa) procurada na situação, por meio da explicação do procedimento de resolução. Além disso, nesse momento se enunciam as propriedades ou afirmações que integram os elementos tecnológicos que explicam ou justificam a técnica sistematizada.

Figura 1: Exercício para introduzir a noção de expressões algébricas Fonte: Iracema; Dulce (1998, p.112)

- utilizando uma tabela:

\begin{tabular}{|l|c|c|c|c|c|}
\hline Horas & 1 & 2 & 3 & 4 & $\ldots$ \\
\hline $\begin{array}{l}\text { Número de } \\
\text { pacotes }\end{array}$ & 60 & 120 & 180 & 240 & $\ldots$ \\
\hline
\end{tabular}

O segundo momento é destinado à avaliação dos elementos técnico-tecnológicos, ocorrendo de forma implícita nos enunciados.

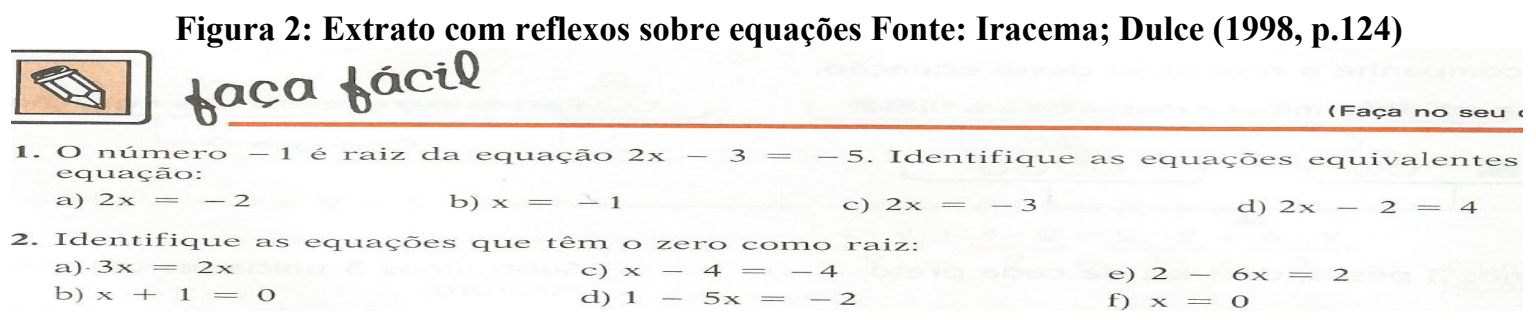

O terceiro momento é dedicado ao trabalho da técnica, indicado nas seções intituladas faça fácil e faça exercícios complementares. Concluímos que nessa coleção a passagem de procedimentos aritméticos para procedimentos algébricos não é realizada de forma explícita, posto que as autoras afirmam que há as regras práticas(técnicas) principais que podem ser agrupados para resolver equações. As autoras não deixam claro quais tipos de equações podem ser resolvidos utilizando-se das operações inversas e quais tipos só podem ser resolvidos efetuando a mesma operação nos dois membros da equação.

Na coleção Ideias e Desafios a transposição das praxeologias matemáticas existentes em volta dos subtipos de tarefas referentes à resolução de equações do $1^{\circ}$ grau se dão por meio de três momentos: o primeiro momento introdução de um problema ou uma situação realizada para formar ou sistematizar a técnica eletiva para resolver a equação (subtipo de tarefa) procurada na situação, por meio de uma explicação do procedimento de resolução. No entanto, é nesse momento que se enunciam as propriedades ou afirmações que integram os elementos tecnológicos que explicam ou justificam a técnica sistematizada.

Figura 3: Exercício para introduzir a noção de expressões algébricas Fonte: Iracema; Dulce (2012, p.147)
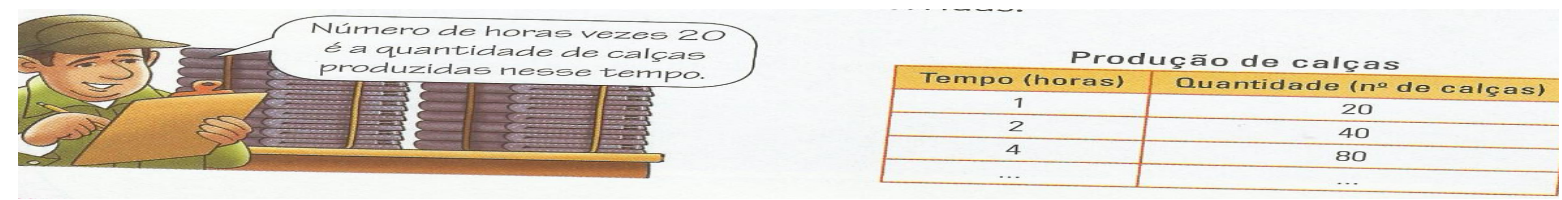
O segundo momento é destinado a avaliação dos elementos técnico-tecnológicos que surgem na situação e ocorrem nas seções denominadas troque ideias e resolva. Assim, nesse momento o aluno tem chance de participar de maneira significativa de sua aprendizagem, pois é nele que as autoras apresentam questionamentos que permitem ao aluno fazer indagações sobre os conceitos e procedimentos explorados no momento anterior.

Figura 4: Extrato com reflexos sobre equações Fonte: Iracema; Dulce (2012, p.164)

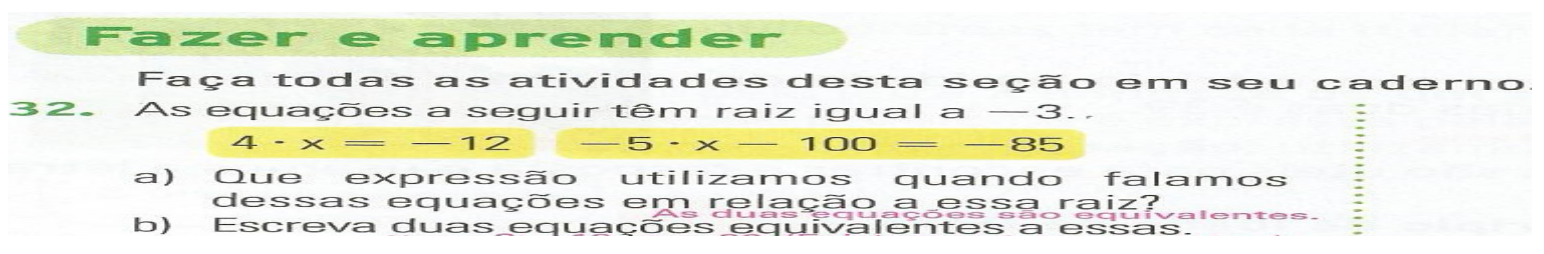

O terceiro momento é dedicado ao trabalho da técnica, indicado nas seções exercícios; exercícios complementares e problemas.

Concluímos que a transposição dos procedimentos aritméticos para os procedimentos algébricos não são realizados de forma explícita nessa coleção. As autoras indicam dois processos (técnicas): o processo geral para resolução de equações em que adota procedimentos para encontrar a raiz da equação e o outro processo em que a regra prática resumiria as etapas, isto é, isolar o x para o $1^{\circ}$ membro invertendo os sinais dos coeficientes ou incógnitas.

No que concerne à organização didática, o mesmo se dá em dois momentos didáticos. $\mathrm{O}$ primeiro, denominado de elaboração e sistematização das técnicas eleitas para resolver equações (subtipos de tarefas) exploradas nas situações introdutórias que se realizam por meio da explicação do processo de resolução. É nesse momento que se enunciam as propriedades ou afirmações que constituem os elementos tecnológicos que explicam ou justificam as técnicas sistematizadas. O segundo, denominado momento do trabalho das técnicas, ocorre através da realização de exercícios apresentados logo em seguida ao processo de sistematização:

Gráfico 1: Comparativos Subtipos de Tarefas Coleção Para Aprender Matemática e Ideias e Desafios

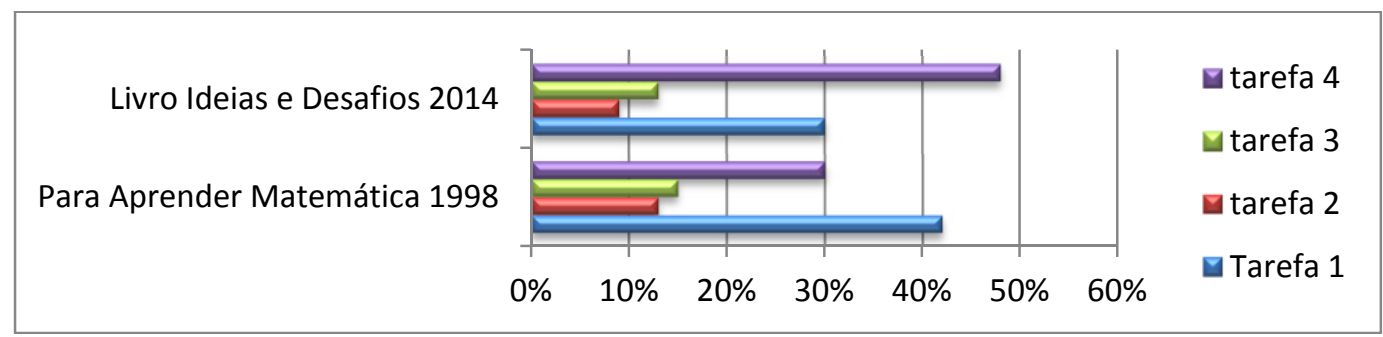


Na coleção Para Aprender Matemática a técnica priorizada foi $\mathrm{t}_{1}$ com $42 \%$, ou seja, equações do tipo $a x+b=c$. Por exemplo $x+8=26$. A coleção Ideias e Desafios de 2014 sofreu alteração em relação a de 1998. A técnica priorizada foi $t_{4}$, com $48 \%$ equações do tipo $A_{1}(x)=A_{2}(x)$. Por exemplo: $3(x-2)+x=\frac{x+5}{2}$.

Em relação ao uso das tecnologias, a coleção Para aprender Matemática concentrava-se na tecnologia da propriedade Princípios de equivalência entre equações, com 32\%. Em 2014, a coleção Ideias e Desafios alterou as prioridades das tecnologias, priorizando as propriedades distributivas da multiplicação/propriedades gerais inversa com $61 \%$.

Alçamos ainda que a coleção de 2014 faz uso de capítulo intitulado de equações, geometria e medidas, como preconiza os Parâmetros Curriculares Nacionais a ligação entre os blocos de conteúdos.

Podemos enfatizar que na coleção Ideias e Desafios houve uma redução significativa em relação ao número de equações prontas para serem resolvidas que em 1998 foi na ordem de 47\% e em 2014 esse percentual foi de 35\%, como mostra o Gráfico 2:

Gráfico 2: Comparativo de equações nas coleções Para Aprender Matemática e Ideias e Desafios

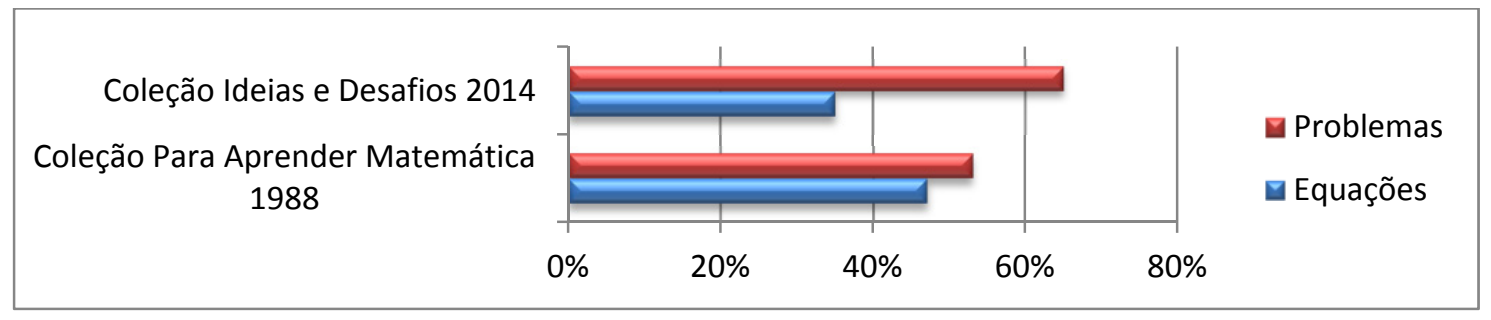

Ainda inferimos que as coleções passaram por mudanças no tocante ao quantitativo de exercícios. Assim, percebemos como positivo a mudança de enfoque na medida em que os alunos não recebem as equações prontas apenas para encontrarem o valor desconhecido (incógnita).

\section{Considerações finais}

Tomando como referência uma coleção aprovada no PNLD de 2014 e outra coleção anterior a avaliação do PNLD, ambas das mesmas autoras, nos permitiu concluir que ao longo desses 15 anos a coleção sofreu modificações significativas em sua estrutura, bem como o avanço da parte da diagramação. 
As coleções analisadas desenvolvem trabalhos de elaboração e sistematização de diferentes técnicas para realizar os diferentes subtipos de tarefas relativos à resolução de equações do $1^{\circ}$ grau. Todavia, tais coleções não justificam a existência dessas diferentes técnicas, assim, não deixam claro os limites ou potencialidades de cada técnica, além de não esclarecerem a distinção entre procedimentos aritméticos e algébricos (CHEVALLARD, 1984).

As transposições didáticas realizadas nessas coleções relativas ao conceito de equação do $1^{\circ}$ grau falham em não deixar claro a transição dos métodos de resolução aritméticos para os métodos de resolução algébricos, assim como não realizaram adequadamente a passagem da Aritmética para Álgebra, como também apontou Araujo (2009). As coleções Para Aprender Matemática e Ideias e Desafios definem equação do primeiro grau como sendo igualdades que contêm letras representando números desconhecidos, denominadas incógnitas, isto é, igualdades entre expressões algébricas. Em ambas as coleções o uso da metáfora da balança de dois pratos é utilizado nas demonstrações das técnicas, de modo que é constante o uso desse recurso.

Por fim, verificamos que as coleções não modificaram as praxeologias matemáticas ao longo dos anos. Contudo, percebemos que as autoras modificaram suas coleções em relação às praxeologias didáticas.

\section{Referências}

ARAUJO, A. J. de, O ensino de Álgebra no Brasil e na França: um estudo sobre o ensino de equações do $1^{\circ}$ grau à luz da teoria antropológica do didático. Tese de doutorado, UFPE, 2009.

BARBOSA E. J. T.; LINS A. F. (Bibi Lins). Equação do Primeiro Grau: um estudo das organizações matemática e didática. In: Anais do XIII Conferência Interamericana de Educação Matemática. Recife, PE, junho de 2011.

BEDNARDZ, N., KIERAN, C. et LEE, L. Approaches to Algebra: Perspectives for Research and Teaching. Mathematics Education Library. Kluwer Academic Peblisshers, London, 1996.

BERNARD, J.E.; COHEN, M.P. Uma integração dos Métodos de Resolução de Equações numa Seqüência evolutiva de Aprendizagem. In: COXFORD, Arthur F. e SHULTE, Albert P. As Idéias da Álgebra. São Paulo: Atual, 1995. Parte 3: Álgebra: Idéias e Questões, Cap. 10, p. 111-126.

BOYER, C. B. História da Matemática. $2^{\circ}$ edição - tradução: Elza F. Gomide, Editora: Editora Edgard Blücher, 1996. 
BOUJADDI, M. Algèbre et généralisation en classe de seconde: 'à chacun sa vérité'. Mémorie profissionnel: I.U.F.M. de Grenable, 1996.

BRASIL. Ministério da Educação. Secretaria de Educação Infantil e Ensino Fundamental. Guia de Livros Didáticos. Brasília, DF, 2010, v.3, $6^{\circ}$ a $9^{\circ}$ séries. 96 p. Secretaria de Educação Fundamental. Parâmetros Curriculares Nacionais (5 a 8 ${ }^{\text {a }}$ Séries) Matemática. Brasília, DF, 1998. 142 p.

BRITO MENEZES, A.P.A.. Contrato Didático e Transposição Didática: InterRelações entre os Fenômenos Didáticos na Iniciação á Álgebra na $6^{\circ}$ Série do Ensino Fundamental. Tese de Doutorado, UFPE, 2006.

CALADO, J.J.G. Compêndio de Álgebra. Lisboa: Livraria Popular de Francisco Franco, 1952.

CHEVALLARD, Yves, BOSCH, Mariana, GASCÓN, Josep. Estudar matemáticas: o elo perdido entre o ensino e a aprendizagem. Tradução: Daisy Vaz de Moraes, Porto Alegre: Artmed Editora, 2001.

L'analyse des pratiques enseignantes en Théorie Anthropologie Didactique. In : Recherches en Didactiques des Mathématiques, 1999. p. 221-266.

. Le passage de l'arithmétique à l'algébrique dans l'enseignement des mathématiques au collège: l'évolution de la transposition didactique. In : Petit X nº 5, IREM, Grenoble, 1984.

Organiser l'etude 1. Structures et Fonctions, in Dorier, J - L. Et al (eds) Actes de la 1 lieme Ecole d'ete de didactique des mathematiques - corps -21-30 Aout 2001, Grenoble : La Pensée Sauvage, pp 3-22.

Le passage de l'arithmetique a l'algebre dans l'enseignement des mathematiques au college. Troisieme partie. Petit $x n^{\circ}$ 30, IREM de Grenoble, pp.5-38, 1990.

- Analyse des pratiques enseignantes et didactique des mathematiques: L'approche anthropologique. Actes de l'U.E. de la Rochelle, 1998.

COSTA, A. A.; DOS Anjos, A. O. Compêndio de matemática. Porto Editora, 1970.

FIORENTINI D; MIORIM, M. A; MIGUEL, A. As concepções de educação algébrica. ProPosições. São Paulo: Cortez, 19 mar, v.1, n.1, p.39-54, 1993.

GARCIA, F. F. Aspectos históricos del paso de la aritmética al álgebra. In: Revista de Didáctica de las Matemáticas. Número 14, ano IV, outubro de 1997. Barcelona: Graó.

GERMI, P. E. Statut des lettres et notion de variable. Petit x, número 45, p. 59-79. Grenoble/França, 1997.

GUELLI, O. Equação: O Idioma da Álgebra. Contando a História da Álgebra. São Paulo SP, Editora Ática, 2005. 
LEE, L. (1996). An initiation into algebraic culture through generalization activities. Em: BEDNARZ, N.; KIERAN,C. \& LEE, L. (Eds.) (1996). Approaches to Algebra:Perspectives of Recearch and Teaching. Dordrecht, Netherlands: Kluwer Academic Publishers, 87-106.

LINS, R. C; GIMENEZ, J. Perspectivas em Aritmética e Álgebra para o Século XXI. 4 ed. Campinas: Papirus Editora, 1997, 176 p.

MIGUEL, FIORENTINI e MIORIM. Álgebra ou Geometria: para onde Pende o Pêndulo?, Pró-posições, vol. 3, n 1, Campinas, SP, 1992.

MIORIN, Â; MIGUEL, A e FIORENTINI, D. Ressonâncias e dissonâncias do movimento pendular entre álgebra e geometria no currículo escolar brasileiro. Zetetiké - n ${ }^{\circ}$, UNICAMP, Campinas, SP, 1993.

MORI,I. Matemática: idéias e desafios. Iracema \& Dulce. Obra em 4 v. para alunos de $5^{\text {a a }}$ $8^{\mathrm{a}}$ séries. São Paulo: Saraiva, $6^{\mathrm{a}}$ ed. 1998.

Matemática: ideias e desafios. Iracema \& Dulce. Obra em 4 v. para alunos de $5^{\mathrm{a}}$ a $8^{\mathrm{a}}$ séries. São Paulo: Saraiva, 17 ed. Reformulada- 2012.

SOUZA, E. R; DINIZ, M. I. S. V. Álgebra: das variáveis às equações e funções. 2 ed. São Paulo: IME-USP, 1996. 111 p. 